\title{
Modified three-step iteration process with errors for three Local strongly pseudo contractive operators
}

\author{
Salwa Salman $\mathrm{Ab}^{1}$ Zena Hussein Mabee ${ }^{2}$ \\ Department of Mathematics-Ibn-Al-Haitham College of Education - University of Baghdad
}

\begin{abstract}
The purpose of this paper is to introduce more general modified two step and three step Ishikawa iterative with errors for local strongly pseudo contractive and local strongly accretive mappings which is much more general than the important class of strongly pseudo contractive and strongly accretive mappings.Also, we study the convergence of this iteration for locally mappings in the framework of Banach space. The results presented in this paper improve, generalize of the results of Mogbademn and OlaleauRafq, Yuguang and Fang and others.
\end{abstract}

\section{Introduction and preliminaries:}

Let $\mathrm{X}$ be a real Banach spaces, $X^{*}$ be the dual space on $X$ The normalized duality mapping $J: X \rightarrow 2^{X^{*}}$ is defined by

$$
J(x)=\left\{f \in X^{*}:\left\langle x_{1} f>=\|x\| .\|f\|,\|x\|=\|f\|\right\} \text { for all } x \in X\right.
$$

where $\langle.,$.$\rangle denotes the generalized duality pairing. It is well know that if X$ is an uniformly smooth Banach space, then $J$ is single-valued and it is uniformly continuous on any bonded sub set of . In the sequel we shall denote single-valued normalized duality mapping by $\mathrm{j}$. By means of the normalized duality mapping by $J$. The symbole is $J$ and $\mathrm{F}(\mathrm{T})$ the identity mapping on $X$ and the set of all fixed points of T respectively.

Let us recall the following three iteration processes due to Ishikawa[1],Mann[2] and Xu [3] Let $\mathrm{k}$ be a non- empty convex subset of an arbitrary normal linear space $\mathrm{X}$ and $\mathrm{T}: \mathrm{K} \rightarrow \mathrm{K}$ be an operator.

i. For any given $x_{0} \in K$ the sequence defined by $\left\langle x_{n}\right\rangle$

$$
\begin{aligned}
& x_{n+1}=\left(1-\propto_{n}\right) x_{n}+\propto_{n} T y_{n} \\
& y_{n}=\left(1-\beta_{n}\right) x_{n}+\beta_{n} T x_{n}, n \geq 0
\end{aligned}
$$

Is called the Ishikawa iteration sequence where $\left\langle\alpha_{n}\right\rangle,\left\langle\beta_{n}\right\rangle$ are real sequences in $[0,1]$ satisfying appropriate conditions.

ii. In particular if $\beta_{n}=0$ for all $n \geq 0$ then the sequence $\left\langle x_{n}\right\rangle$ defined by:

$$
x_{n+1}=\left(1-\propto_{n}\right) x_{n}+\propto_{n} T_{1} y_{n}, n \geq 0
$$

Is called the Mann iteration sequence.

iii. For any $x_{0} \in k$ the sequence $\left\langle x_{n}\right\rangle$ defined by:

$$
\begin{gathered}
x_{n+1}=a_{n} x_{n}+b_{n} T y_{n}+c_{n} u_{n} \\
y_{n}=a_{n}^{\prime} x_{n}+b_{n} T y_{n}+\dot{c}_{n} v_{n}, n \geq 0 .
\end{gathered}
$$

Where $\left\langle u_{n}\right\rangle,\left\langle v_{n}\right\rangle$ are arbitrary bounded sequences in $\mathrm{K}$ and $\left\langle a_{n}\right\rangle,\left\langle b_{n}\right\rangle,\left\langle c_{n}\right\rangle$ $\left\langle a_{n}^{\prime}\right\rangle,\left\langle\dot{b}_{n}\right\rangle,\left\langle\dot{c}_{n}\right\rangle$ are real sequences in $[0,1]$ such that $a_{n}+b_{n}+c_{n}$ $=a_{n}^{\prime}+b_{n}+\dot{c}_{n}=1$ for all $n \geq 0$ is called the Ishikawa iteration sequences with errors.

iv. If, with the same notations and definitions as in (iii), $\dot{b}_{n}=\dot{c}_{n}=0$ for all $n \geq 0$ then the sequence $\left\langle x_{n}\right\rangle$ now defined by:

$$
x_{n+1}=a_{n} x_{n}+b_{n} T y_{n}+c_{n} u_{n}, n \geq 0
$$


Is called the Mann iteration sequences with errors.

It is clear that the Ishikawa and Mann iteration sequences are all special cases of the Ishikawa and Mann iteration sequences with errors respectively.

Now Let, $T_{1}, T_{2}: K \rightarrow K{ }^{1}$ be two mappings. For any given $x_{o} \in k$ the more general modified two step iteration $\left\langle x_{n}\right\rangle$ defined by:

$$
\begin{gathered}
x_{n+1}=a_{n} x_{n}+b_{n} T_{1} y_{n}+c_{n} u_{n} \\
y_{n}=a_{n}^{\prime} x_{n}+\dot{b}_{n} T_{2} y_{n}+\dot{c}_{n} v_{n}, n \geq 0
\end{gathered}
$$

Where the read sequence as in (1.2)

It is that the iteration schemes (1.1) - (1.2) are special cases of (1.3).

Noor[4] gave the following three- step iteration process for solving non-linear operator equations in real Banach space, let $\mathrm{K}$ be a nonempty closed convex subset of $\mathrm{X}$ and $T_{1}, T_{2}: K \rightarrow K$ be mapping. For an arbitrary $x_{O} \in k$ be mapping. For an arbitrary the sequence $\left\langle x_{n}\right\rangle$ defined by:

$$
\begin{aligned}
& x_{n+1}=\left(1-\alpha_{n}\right) x_{n}+\propto_{n} T_{1} y_{n} \\
& y_{n}=\left(1-\beta_{n}\right) x_{n}+\beta_{n} T_{2} z_{n} \\
& z_{n}=\left(1-\gamma_{n}\right) x_{n}+\gamma_{n} T_{3} x_{n}, \quad n \geq 0
\end{aligned}
$$

Where $\left\langle\alpha_{n}\right\rangle,\left\langle\beta_{n}\right\rangle,\left\langle\gamma_{n}\right\rangle$ are three sequences in[0,1], is called the three-step iteration or (Noor iteration).

Rafiq[7] introduced the following new type of iteration, the modified three-step iteration $\left\langle x_{n}\right\rangle$ is defined by:

$$
\begin{aligned}
& x_{n+1}=\left(1-\propto_{n}\right) x_{n}+\propto_{n} T y_{n} \\
& y_{n}=\left(1-\beta_{n}\right) x_{n}+\beta_{n} T z_{n} \\
& z_{n}=\left(1-\gamma_{n}\right) x_{n}+\gamma_{n} T_{3} x_{n}, \quad n \geq 0
\end{aligned}
$$

Where $\left\langle\alpha_{n}\right\rangle,\left\langle\beta_{n}\right\rangle,\left\langle\gamma_{n}\right\rangle$ are real sequences in[0,1].

It is clear that the iteration schemes (1.4) is special case of (1.5), we define the more general modified three - step iteration process with errors by:

$x_{n+1}=a_{n} x_{n}+b_{n} T_{1} y_{n}+c_{n} u_{n}$

$y_{n}=a_{n}^{\prime} x_{n}+\dot{b}_{n} T_{2} z_{n}+\dot{c}_{n} v_{n}$

$z_{n}=\dot{a}_{n} x_{n}+\dot{b}_{n} T_{3} y_{n}+\dot{c}_{n} w_{n}, n \geq 0$

Where $\left\langle u_{n}\right\rangle,\left\langle v_{n}\right\rangle$ and $\left\langle w_{n}\right\rangle$ are arbitrary bounded sequences in $\mathrm{k}$ and $\left\langle a_{n}\right\rangle$ $\left\langle b_{n}\right\rangle,\left\langle c_{n}\right\rangle,\left\langle a_{n}^{\prime}\right\rangle,\left\langle\dot{b}_{n}\right\rangle,\left\langle\dot{c}_{n}\right\rangle,\left\langle\dot{a}_{n}\right\rangle,\left\langle\dot{b}_{n}\right\rangle$ and $\left\langle\dot{c}_{n}\right\rangle_{\text {i }}$ are real sequences in $[0,1]$ satisfying some conditions.

Now, we introduce local strongly pseudo -contractive (local strongly accretive) operators as follows.

Definition(1.1)[6],[7]:-

An operator $\mathrm{T}$ with domain $\mathrm{D}(\mathrm{T})$ and $\operatorname{rang} \mathrm{R}(\mathrm{T})$ in $\mathrm{X}$ is called:-1- Pseudo contractive if for all $r>0$, the inequality

$$
\|x-y\| \leq\left\|(1+r)(x-y)-r\left(T_{x}-T_{y}\right)\right\|
$$


2- Accretive if for all $r>0$ the inequality

$$
\|x-y\| \leq\left\|x-y+r\left(T_{x}-T_{y}\right)\right\|
$$

holds for each pair of points $x, y \in D(T)$.

An operator $\mathrm{T}$ is called strongly pseudo-contractive (strongly accretive) if there exists a real number $k \in(0,1)$ such that

(T-KI) is pseudo-contractive (accretive).

3- Local strongly pseudo-contractive if for each $x \in D(T)$ there exists $t_{x}>1$ such that for all $y \in D(T)$ and $r>0$

$$
\|x-y\| \leq\left\|(1+r)(x-y)-r t_{x}\left(T_{x}-T_{y}\right)\right\|
$$

4- local strongly accretive if for given $x \in D(T)$ there exists $k_{x} \in(0,1)$ such that for each $y \in D(T)$ there is

$$
\begin{aligned}
& j(x-y) \in \mathrm{J}(x-y) \text { satisfying } \\
& <T_{x}-T_{y}, j(x-y)>\geq \mathrm{k}_{\mathrm{x}}\|x-y\|^{2}
\end{aligned}
$$

5- strongly pseudo-contractive (strongly accretive) if it is local strongly pseudo-contractive(local strongly accretive) and $t_{x=t} \quad\left(k_{x}=k\right)$ and $\left(k_{x}=k\right)_{\text {independent of }} x \in D(T)$

\section{Remark (1-2):-[7]}

1. Each strongly pseudo contractive operator is local strongly pseudo-contractive and each stronglyaccretive operator is local strongly accretive.

2. $\mathrm{T}$ is local strongly pseudo-contractive if and only if (I-T) is local strongly accretive and $k_{x}=1-\frac{1}{t_{x}}$, where $\mathrm{t}_{\mathrm{x}}$ and $\mathrm{k}_{\mathrm{x}}$ are the constants oppearing in (1.8) and (1.9) respectively.

3. If $\mathrm{T}$ is local strongly accretive then $(T-K x I)$ accretive.

\section{Lemma (1.3)[5]:-}

Let $\mathrm{X}$ be a real Banach space and $\mathrm{J}: X \rightarrow 2^{X^{*}}$ be the normalized duality mapping. Then, for any $\mathrm{x}, \mathrm{y} \in$ $\mathrm{X}$

$$
\|x-y\|^{2} \leq\|x\|^{2}+2<y, j(x+y)>, \forall j(x+y) \in J(x+y)
$$

\section{Lemma (1.4) [8] :-}

Let $\left\langle\alpha_{n}\right\rangle$ be a non-negative sequence which satisfied the following inequality

$\propto_{n+1} \leq\left(1-\lambda_{n}\right) \alpha_{n}+\delta_{n}$

$\sum_{n=1}^{\infty} \lambda_{n}=\infty$ and Where $\lambda_{n} \in(0,1), \forall n \in N$, and $\delta_{n}=0\left(\lambda_{n}\right)$

Then $\lim _{n \rightarrow \infty} \propto_{n}=0$

our purpose in this paper to prove that the modified three-step iteration process with error for three local strongly pseudo-contractive operators strongly convergence to fixed points. The results presented in this paper generalize the corresponding Main Results in[4],[5],[9],[10],[11],[12], and others

\section{Theorem (2.1):-}

Let $\mathrm{X}$ be a uniformly smooth Banach space and $T_{1}, T_{2}: X \rightarrow X_{\text {be a local strongly accretive mapping }}$ suppose that there exists a solution of the equation $T_{i} x=f(i=1,2)$ for some $f \in X$ define $H_{i} .: X \rightarrow X$ by $H_{i} x=f+x-T_{i} x$ suppose that $R(X)$ is bounded.

Let $\chi_{0} \in X$ the two step iteration sequence with errors $<x_{n}>$ defined by:

$$
\begin{aligned}
& x_{n+1}=a_{n} x_{n}+b_{n} H_{1} y_{n}+c_{n} u_{n} \\
& y_{n}=a_{n}^{\prime} x_{n}+\dot{b}_{n} H_{2} x_{n}+\dot{c}_{n} v_{n}
\end{aligned}
$$


Where $\left\langle u_{n}\right\rangle$ and $\left\langle v_{n}\right\rangle$ are two bounded sequence in $\mathrm{X}$ and $\left\langle a_{n}\right\rangle,\left\langle b_{n}\right\rangle,\left\langle b_{n}\right\rangle,\left\langle c_{n}\right\rangle$ and $\left.k c_{n}\right\rangle$ are real sequences in $[0,1]$ such that $a_{n}+b_{n}+c_{n}=a_{n}+b_{n}+\dot{c}_{n}=1_{\text {satisfying the conditions:- }}$

i) $\lim _{n \rightarrow \infty} b_{n}=\lim _{n \rightarrow \infty} b_{n}=0$

ii) $\sum_{n=0}^{\infty} b_{n}=\infty$,

iii) $c_{n} \leq b_{n}, \dot{c}_{n} \leq \dot{b}_{n}$.

Then the sequence $\left\langle x_{n}>\right.$ converges strongly to the unique fixed point of the equation $T_{i} x=f$

\section{Proof:-}

Let $T_{i} w=f$ so that $\mathrm{w}$ is a fixed point of $H_{i}$, since $T_{i}$ is local strongly accretive mapping, it follows from definition of $H_{i} \quad(\mathrm{i}=1,2)$ that

$$
<H_{i} x-H_{i} y, j(x-y)>\leq\|x-y\|^{2}-\|x-y\|^{2}
$$

Setting $\mathrm{y}=\mathrm{w}$, we have

$$
<H_{i} x-H_{i} w, j(x-y)>\leq\|x-y\|^{2}-\|x-w\|^{2}
$$

If $\mathrm{z}$ is a fixed point of $H_{i}$, then (4) with $\mathrm{x}=\mathrm{z}$ implies $\mathrm{w}=\mathrm{z}$, we prove that $\left\langle x_{n}\right\rangle$ and $\left\langle y_{n}\right\rangle$ are bounded.

Let $\sup \left\{\left\|H_{i} x-H_{i} w\right\|+\left\|H_{i} y-w\right\|: n \geq 0\right\}+\left\|x_{0}-w\right\|=A_{i}$

$\sup \left\{\left\|u_{n}\right\|+\left\|v_{n}\right\|: n \geq 0\right\}=B, M_{i}=A_{i}+B$ for all $\mathrm{i}=1,2$

by (1) and (iii) we get

$$
\text { and } M=\sup \left\{M_{1}, M_{2}\right\}
$$

$$
\begin{aligned}
\left\|x_{n+1}-w\right\| & \leq a_{n}\left\|x_{n}-w\right\|+b_{n}\left\|H_{1} y_{n}-w\right\|+c_{n}\left\|u_{n}\right\| \\
& \leq a_{n}\left\|x_{n}-w\right\|+b_{n} A_{1}+b_{n} B \\
& \leq a_{n}\left\|x_{n}-w\right\|+b_{n} M_{1} \\
& \leq a_{n}\left\|x_{n}-w\right\|+b_{n} M
\end{aligned}
$$

Now, from(2) and(iii), we get

$\left\|y_{n}-w\right\| \leq a_{n}\left\|x_{n}-w\right\|+b_{n}\left\|H_{2} x_{n}-w\right\|+\dot{c}_{n}\left\|v_{n}\right\|$

$$
\begin{aligned}
& \leq \dot{a}_{n}\left\|x_{n}-w\right\|+\dot{b}_{n} A_{2}+\dot{b}_{n} B \\
\left\|y_{n}-w\right\| & \leq \dot{a}_{n}\left\|x_{n}-w\right\|+\dot{b}_{n} M_{2} \\
& \leq \dot{a}_{n}\left\|x_{n}-w\right\|+\dot{b}_{n} M
\end{aligned}
$$

Now, we show by induction that $\left\|x_{n}-w\right\| \leq M$

For all $\geq 0$ for $\mathrm{n}=0$ we have $\left\|x_{o}-w\right\| \leq A_{i} \leq M_{i} \leq M$

Assume now that $\left\|x_{n}-w\right\| \leq M$ for some $n \geq 0$. then by(5)

$$
\begin{gathered}
\left\|x_{n+1}-w\right\| \leq a_{n}\left\|x_{n}-w\right\|+b_{n} M \\
\quad \leq a_{n} M+b_{n} M \\
=\left(a_{n}+b_{n}\right) M=\left(1-c_{n}\right) M \leq M
\end{gathered}
$$

Therefore; the inequality (7) holds

Substrituting (7) into(6), we get

$$
\left\|y_{n}-w\right\| \leq M
$$


From (6), we have

$\left\|y_{n}-w\right\|^{2} \leq a^{2}{ }_{n}\left\|x_{n}-w\right\|^{2}+2 a_{n} \dot{b}_{n} M\left\|x_{n}-w\right\|+b^{2}{ }_{n} M^{2}$

Since $a_{n} \leq 1$ and $\left\|x_{n}-w\right\| \leq M$, we get

$\left\|y_{n}-w\right\|^{2} \leq\left\|x_{n}-w\right\|^{2}+2 \dot{b}_{n} M^{2}+\dot{b}_{n} M^{2}$

$$
=\left\|x_{n}-w\right\|^{2}+3 b_{n} M^{2}
$$

Using Lemma(1.3), we get

$\left\|x_{n+1}-w\right\|^{2} \leq\left\|a_{n}\left(x_{n}-w\right)+c_{n} u_{n}+b_{n}\left(H_{1} y_{n}-w\right)\right\|^{2}$

$\leq\left\|a_{n}\left(x_{n}-w\right)+c_{n} u_{n}\right\|^{2}+2 b_{n}<H_{1} y_{n}-w, j\left(x_{n+1}-w\right)>$

$\leq a_{n}^{2}\left\|x_{n}-w\right\|^{2}+2 a_{n} c_{n}\left\|u_{n}\right\|\left\|x_{n}-w\right\|+c_{n}^{2}\left\|u_{n}\right\|^{2}+2 b_{n}$ $<H_{1} y_{n}-w, j\left(y_{n}-w\right)>+2 b_{n}<H_{1} y_{n}-w, j\left(x_{n+1}-w\right)-j\left(y_{n}-w\right)$

$>$

Hence, using definition of local strongly accretive and definition of $\mathrm{M}$, we get

$\left\|x_{n+1}-w\right\|^{2} \leq\left\|x_{n}-w\right\|^{2}-2 b_{n}\left\|x_{n}-w\right\|^{2}+b_{n}{ }^{2}\left\|x_{n}-w\right\|^{2}+2 a_{n} c_{n} M^{2}+$ $c_{n}^{2} M^{2}+2 b_{n}\left\|y_{n}-w\right\|^{2}-2 b_{n} k_{x}\left\|y_{n}-w\right\|^{2}+2 b_{n} e_{n}$

Where $e_{n}=<H_{1} y_{n}-w, j\left(x_{n+1}-w\right)-j\left(y_{n}-w\right)>$

By (7), (9), $c_{n} \leq b_{n}$ and $-2 a_{n} c_{n}+c_{n}{ }^{2} \leq 0$, we abtain

$\left\|x_{n+1}-w\right\|^{2} \leq\left\|x_{n}-w\right\|^{2}-2 b_{n} M^{2}+b_{n}{ }^{2} M^{2}+2 a_{n} c_{n} M^{2}-c_{n}{ }^{2} M^{2}+2 c_{n}{ }^{2} M+$ $2 b_{n}\left(M^{2}+2 b_{n} M^{2}\right)-2 b_{n} k_{x}\left\|y_{n}-w\right\|^{2}+2 b_{n} e_{n}$

$\leq\left\|x_{n}-w\right\|^{2}-b_{n} M^{2}+b_{n}{ }^{2} M^{2}-\left(-2 a_{n} c_{n}-c_{n}{ }^{2}\right) M^{2}+2 c_{n}-b_{n} M^{2}+2 b_{n} M^{2}+$ $4 b_{n} . b_{n} M^{2}-2 b_{n} k_{x}\left\|y_{n}-w\right\|^{2}+2 b_{n} e_{n}$

$$
=\left\|x_{n}-w\right\|^{2}-2 b_{n} k_{x}\left\|y_{n}-w\right\|^{2}+b_{n} \lambda_{n}
$$

Where

$\lambda_{n}=\left(b_{n}+2 c_{n}+4 \dot{b}_{n}\right) M^{2}+2 e_{n}$

First, we show that $c_{n}^{-} 0$ as $n \rightarrow \infty$.

From (1) and(2) we get

$\left\|x_{n+1}-y_{n}\right\| \leq \|\left(a_{n}-a_{n}\right)\left(x_{n}-w\right)+b_{n}\left(H_{1} y_{n}-w\right)-b_{n}\left(H_{2} x_{n}-w\right)+c_{n} u_{n}-$ $\dot{c}_{n} \hat{v}_{n}$

$\leq\left(a_{n}-a_{n}\right)\left\|x_{n}-w\right\|+b_{n}\left\|H_{1} y_{n}-w\right\|+b_{n}\left\|H_{2} x_{n}-w\right\|+c_{n}\left\|u_{n}\right\|+\dot{c}_{n} \|$ $v_{n} \|$

$\leq\left(1-b_{n}-c_{n}-1+\dot{b}_{n}+\dot{c}_{n}\right)\left\|x_{n}-w\right\|+b_{n}\left\|H_{1} y_{n}-\right\|+\dot{b}_{n} \| H_{2} x_{n}-w$

$\left\|+b_{n}\right\| u_{n}\left\|+\dot{b}_{n}\right\| v_{n} \|$

By (7) and definition of M. we get

$\left\|x_{n+1}-y_{n}\right\| \leq 2\left(b_{n}+\ddot{b}_{n}\right) M+\left(b_{n}+\dot{b}_{n}\right) M+\left(b_{n}+\dot{b}_{n}\right) M_{\text {i.e., }}$

$$
\left\|x_{n+1}-y_{n}\right\| \leq 4\left(b_{n}-\dot{b}_{n}\right) M
$$

Therefore; $\left\|x_{n+1}-w-\left(y_{n}-w\right)\right\| \rightarrow 0$ as $n \rightarrow \infty$.

Since $\left\langle x_{n+1}-y_{n}\right\rangle,\left\langle y_{n}-w\right\rangle$ and $\left\langle H_{1} y_{n}-w\right\rangle$ are bounded and $\mathrm{j}$ is uniformly continuous on any bounded subset of $X$ we have 
Thus, $\lim _{n \rightarrow \infty} \lambda_{n}=0$

$$
j\left(x_{n+1}-w\right)-j\left(y_{n}-w\right) \rightarrow 0 \text { and }<e_{n}>\rightarrow 0 \text { as } n \rightarrow \infty .
$$

Let $\delta=\inf \left\{\left\|y_{n}-w\right\|^{2}: n \geq 0\right.$

We prove that $\delta=0$

Assume the contrary, $\delta>0$,

Then $\left\|y_{n}-w\right\|^{2} \geq \delta>0$ for all $\geq 0$, hence

$k_{x}\left(\left\|y_{n}-w\right\|^{2}\right) \geq k_{x}(\delta)>0$ where $k_{x} \in(0,1)$

Thus from(11)

$$
\left\|x_{n+1}-w\right\|^{2} \leq\left\|x_{n}-w\right\|^{2}-b_{n} k_{x}(\delta)-b_{n}\left[k_{x}(\delta)-\lambda_{n}\right]
$$

Since $\lim _{n \rightarrow \infty} \lambda_{n}=0$, there exists a positive integerno

Such that $\lambda_{n} \leq k_{x}(\delta)$ for all $n \geq n_{o}$

Therefore, from(13), we have

$$
\begin{aligned}
& \left\|x_{n+1}-w\right\|^{2} \leq\left\|x_{n}-w\right\|^{2}-b_{n} k_{x}(\delta) \quad \text { or } \\
& b_{n} k_{x}(\delta) \leq\left\|x_{n}-w\right\|^{2}-\left\|x_{n+1}-w\right\|^{2} \quad \text { for all } n \geq 0
\end{aligned}
$$

Hence.

$k_{x}(\delta) \sum_{j=n_{o}}^{n} b j=\left\|x_{n_{0}}-w\right\|^{2}+\left\|x_{n+1}-w\right\|^{2} \leq\left\|x_{n_{0}}-w\right\|^{2}$.

Which implies $\sum_{n=0}^{\infty} b_{n}<\infty$ contradicting (ii). Therefore $\delta=0$ from definition of $\delta_{\text {; }}$, there exists a subsequence of $<\left\|y_{n}-w\right\|>_{\text {which we will denote by }}<\left\|y_{i}-w\right\|>$ such that

$$
\lim _{n \rightarrow \infty}\left\|\dot{y}_{i}-w\right\|=0
$$

$\left\|x_{n}-w\right\|^{2} \leq\left\|y_{n}-w+\left(\dot{b}_{n}+\dot{c}_{n}\right)\left(x_{n}-w\right)-\dot{b}_{n}\left(H_{2} y_{n}-w\right)-\dot{c}_{n} v_{n}\right\| \leq \| y_{n}-$

$w\left\|+\left(\dot{b}_{n}+\dot{c}_{n}\right)\right\|\left(x_{n}-w\right)\left\|-\dot{b}_{n}\right\|\left(H_{2} x_{n}-w\right) \|$

$+\dot{c}_{n}\left\|v_{n}\right\|$.

Since $\dot{c}_{n} \leq \dot{b}_{n}$ and by definition $A_{i}, B$ and $\mathrm{M}$, we get

$\left\|x_{n}-w\right\|^{2} \leq\left\|y_{n}-w\right\|+2 b_{n} M$ for all $n \geq 0$

Thus

$$
\lim _{j \rightarrow \infty}\left\|x_{j}-w\right\|=0
$$

Now, let $\in>0$ be arbitrary, since $\lim _{n \rightarrow \infty} b_{n}=\lim _{n \rightarrow \infty} b_{n}=0$ and $\lim _{n \rightarrow \infty} \lambda_{n}=0$ there exists a positive integer. no.

Such that

$$
b_{n} \leq \frac{\epsilon}{12 M} \quad, \quad \dot{b}_{n} \leq \frac{\epsilon}{12 M} \quad, \lambda_{n} \leq k_{x}\left(\frac{\epsilon}{3}\right) \text { for all } n \geq N_{0}
$$

From(16), there exists $k \geq N_{0}$ such that

$$
\left\|x_{k}-w\right\|<\epsilon
$$

We prove by induction that $\left\|x_{k+n}-w\right\|<\in$ for all $n \geq 0$

Suppose that (18) holds for some $n \geq 0$ and that

For $\mathrm{n}=0$, we see that (18) holds by (17) 
$\left\|x_{k+n+1}-w\right\| \geq \in$. then by(12), we get

$$
\begin{aligned}
\in \leq \| x_{k+n+1}- & w\|=\| y_{k+n}-w+x_{k+n+1}-y_{k+n} \| \\
& \leq\left\|y_{k+n}-w\right\|+\left\|x_{k+n+1}-y_{k+n}\right\| \\
& \leq\left\|y_{k+n}-w\right\|+3\left(b_{k+n}+b_{k+n}\right) M \\
& \leq\left\|y_{k+n}-w\right\|+\frac{\epsilon}{2}
\end{aligned}
$$

Hence, $\left\|y_{k+n}-w\right\| \geq \frac{\epsilon}{2}$

From(11), we get

$$
\epsilon^{2} \leq\left\|x_{k+n+1}-w\right\|^{2} \leq\left\|x_{k+n}-w\right\|^{2}-2 b_{k+n} k_{x}\left(\frac{\epsilon}{3}\right)+\quad b_{k+n} k_{x}
$$

$\leq\left\|x_{k+n}-w\right\|^{2}<\epsilon^{2}$, which is a contradiction

Thus we proved (18), hence, $\lim _{n \rightarrow \infty}\left\|x_{n}-w\right\|=0$

\section{Theorem (2.2):-}

Let $\mathrm{X}$ be a uniformly smooth Banach space, let $\mathrm{k}$ be a non empty bounded closed subset of $\mathrm{X}$ and $T_{1}, T_{2}: K \rightarrow K$ be local strongly pseudo-contractive mappings. Let $\mathrm{w}$ be a fixed point of $T_{i}(\mathrm{i}=1,2)$ and let $x_{0} \in k$ the Ishikawa iteration sequence $<x_{n}>$ be defined by

$$
\begin{aligned}
& x_{n+1}=a_{n} x_{n}+b_{n} T_{1} y_{n}+c_{n} u_{n} \\
& y_{n}=\dot{a}_{n} x_{n}+\dot{b}_{n} T_{2} y_{n}+\dot{c}_{n} v_{n}, n \geq 0
\end{aligned}
$$

Where $\left\langle u_{n}\right\rangle$ and $\left\langle v_{n}\right\rangle \subset K,\left\langle b_{n}\right\rangle,\left\langle b_{n}\right\rangle,\left\langle c_{n}\right\rangle,\left\langle c_{n}\right\rangle$ are sequences as in theorem (2.1). then $<x_{n}>$ converges strongly to the unique fixed point of $T_{i}$.

\section{Proof:-}

Obviously $\left\langle x_{n}>\right.$ and $\left\langle y_{n}>\right.$ are both contained in $\mathrm{K}$ and therefore, bounded

Since $T_{i}$ is local strongly pseudo-contractive, then (I - $T_{i}$ ) is local strongly accretive for all $(\mathrm{i}=1,2)$ put $\mathrm{y}=\mathrm{w}$ and $\left(T_{i}=H_{i}\right)$, we get $(7)$ the proof of theorem (2.1) follows.

Now, we establish the convergence of more general modified three-step to the unique solution of uniformly continuous and locally operators in arbitrary Banach space.

\section{Theorem (2.3):-}

Let $\mathrm{X}$ be a uniformly smooth Basnch space, let $\mathrm{k}$ be a non empty bounded closed subset of $\mathrm{X}$ $(\mathrm{I}=1,2,3)$ and $T_{i}(\mathrm{i}=1,2,3)$ is a local strongly pseudo-contractive mappings of $\mathrm{k}$ and $\bigcap_{i=1}^{3} F\left(T_{i}\right) \neq \emptyset$. Define sequence $<x_{n}>$ iteratively for $x_{1} \in k$ by

$x_{n+1}=a_{n} x_{n}+b_{n} T_{1} y_{n}+c_{n} u_{n}$

$y_{n}=\dot{a}_{n} x_{n}+\dot{b}_{n} T_{2} Z_{n}+\dot{c}_{n} v_{n}$

$z_{n}=\dot{a}_{n} x_{n}+\dot{b}_{n} T_{3} y_{n}+\dot{c}_{n} w_{n}$

Where $\left\langle u_{n}\right\rangle,\left\langle v_{n}\right\rangle$ and $\left\langle w_{n}\right\rangle$ are, bounded sequence in $\mathrm{k}$ and $\left\langle a_{n}\right\rangle,\left\langle\hat{a}_{n}\right\rangle,\left\langle\hat{a}_{n}\right\rangle,\left\langle b_{n}\right\rangle,\left\langle\hat{b}_{n}\right\rangle,\left\langle\hat{b}_{n}\right\rangle$,

$\left\langle c_{n}\right\rangle,\left\langle\dot{c}_{n}\right\rangle$ and $\left\langle\dot{c}_{n}\right\rangle$ are real sequence in [0.1] such that

$a_{n}+b_{n}+c_{n}=\dot{a}_{n}+\dot{b}_{n}+\dot{c}_{n}=\dot{a}_{n}+\dot{b}_{n}+\dot{c}_{n}=1$ and satisfying the following:

$-\sum b_{n}=\infty$

- $\lim _{n \rightarrow \infty} b_{n}=\lim _{n \rightarrow \infty} \hat{b}_{n}=\lim _{n \rightarrow \infty} \hat{b}_{n}=0$

$-\propto_{n}=b_{n}+c_{n}, \beta_{n}=\dot{b}_{n}+\dot{c}_{n}, \gamma_{n}=\dot{b}_{n}+\dot{c}_{n}$ and $\lim _{\mathrm{n} \rightarrow \infty} \frac{1}{1+k_{x} \propto_{n}}=0$

For all $k_{x} \in(0,1)$ Then the sequence $<x_{n}>$ converges strongly to the unique a fixed point of $T_{i}$ 


\section{Proof:-}

Since $\bigcap_{i=1}^{3} F\left(T_{i}\right) \neq \emptyset$ it follows from(1.8) that $\bigcap_{i=1}^{3} F\left(T_{i}\right)$ is singleton sag q . The operators $T_{i}$ is local strongly pseudo contractive implies that $\left(I-T_{i}\right)$ is local strongly accretive and therefore $\left(I-T_{i}\right)-k_{x} I=I-T_{i}-k_{x} I \quad(i=1,2,3)$ is accretive. Hence, for all $r>0$ and $k_{x} \in(0,1)$, we have:

$\|x-y\| \leq \| x-y+r\left[\left(I-T_{i}-k_{x} I\right) x-\left(I-T_{i}-k_{x} I\right) y\right]$

From our hypothesis, we obtain the following

$$
\begin{gathered}
x_{n+1}=\left(1-\propto_{n}\right) x_{n}+b_{n} T_{1} y_{n}+c_{n} u_{n} \\
=x_{n}-\propto_{n} x_{n}+b_{n} T_{1} y_{n}+c_{n} u_{n}
\end{gathered}
$$

Now, we have

$$
\begin{aligned}
& x_{n}=x_{n+1}+\propto_{n} x_{n}-b_{n} T_{1} y_{n}-c_{n} u_{n} \\
& x_{n}=x_{n+1}+\propto_{n} x_{n+1}-\propto_{n} x_{n+1}+\propto_{n} x_{n}-b_{n} T_{1} y_{n}-c_{n} u_{n} \\
& =\left(1+\propto_{n}\right) x_{n+1}+\propto_{n}\left(I-T_{i}-k_{x} I\right) x_{n+1}-\propto_{n}\left(I-T_{i}-k_{x} I\right) x_{n+1} \\
& -\propto_{n}\left(x_{n+1}-x_{n}\right)-b_{n} T_{1} y_{n}-c_{n} u_{n} \\
& =\left(1+\propto_{n}\right) x_{n+1}+\propto_{n}\left(I-T_{i}-k_{x} I\right) x_{n+1}-\propto_{n}\left(I-k_{x} I\right) x_{n+1} \\
& +\propto_{n} T_{i x_{n+1}}+\propto_{n}\left(x_{n}-x_{n+1}\right)-b_{n} T_{1} y_{n}-c_{n} u_{n}
\end{aligned}
$$

Since $\mathrm{q}$ is a fixed point of $T_{i}$, then

$$
q=\left(1+\propto_{n}\right) q+\propto_{n}\left(I-T_{i}-k_{x} I\right) q-\propto_{n}\left(I-k_{x}\right) q
$$

Subtracting(20) from(19) we obtain

$$
\begin{aligned}
& x_{n}-q=\left(1+\propto_{n}\right)\left[\left(x_{n+1}-q\right)+\frac{\propto_{n}}{1+\propto_{n}}\left\{\left(I-T_{i}-k_{x}\right) x_{n+1}-\left(I-T_{i}-k_{x} I\right) q\right\}\right]- \\
& \propto_{n}\left(I-k_{x}\right)\left(x_{n+1}-q\right)+\left[\propto_{n}\left(T_{i}-I\right) x_{n+1}\right. \\
& \left.\quad \quad b_{n} T_{1} y_{n}\right]+\left[\propto_{n} x_{n}-c_{n} u_{n}\right]
\end{aligned}
$$

Since $T_{i}$ is local strongly pseudo contractive, then (21) yields

$\left\|x_{n}-q\right\| \geq\left(1+\propto_{n}\right)\left\|x_{n+1}-q\right\|-\propto_{n}\left(I-k_{x}\right)\left\|x_{n+1}-q\right\|-\| \propto_{n}\left(T_{i}-I\right) x_{n+1}$ $c_{n} u_{n}\|-\| \propto_{n} x_{n}-b_{n} T_{1} y_{n} \|$.

$=\left(1-k_{x} \propto_{n}\right)\left\|x_{n+1}-q\right\|-\left\|\propto_{n}\left(T_{i}-I\right) x_{n+1}-c_{n} u_{n}\right\|-\left\|\propto_{n} x_{n}-b_{n} T_{1} y_{n}\right\|$.

$\left\|x_{n}-q\right\| \leq \frac{1}{1+k_{x} \propto_{n}}\left[\left\|x_{n}-q+\right\| \propto_{n}\left(T_{i}-I\right) x_{n+1}-c_{n} u_{n}\|+\| \propto_{n} x_{n}-b_{n} T_{1} y_{n} \|\right]$

Since $\mathrm{T}$ is uniformly continuous on the bounded set $\mathrm{k}$ there exists a positive real number $M<\bowtie$ such that

$$
\left\|\left(x_{n+1}-q\right)\right\| \leq \frac{1}{1+\propto_{n} k_{x}}\left\|x_{n}-q\right\|+\frac{1}{1+\propto_{n} k_{x}} M
$$


Now, put $\delta_{n}=\frac{1}{1+\propto_{n} k_{x}}, \sigma_{n}=\delta_{n} M$ and $q_{n}=\left\|x_{n}-q\right\|$

Thus (22) reduces to

$q_{n+1} \leq \delta_{n} q_{n}+\sigma_{n}$

Since $0 \leq \delta_{n} \leq 1, \lim _{n \rightarrow \infty} \delta_{n}=0$ and $\lim _{n \rightarrow \infty} \sigma_{n}=0$. Therefore by Lemma (1.4), we have $\lim _{n \rightarrow \infty} q_{n}=0$

Which implies that the sequence $<x_{n}>$ converges strongly to q corollary (2.4).

\section{Corollary(2.4):-}

Let $\mathrm{X}$ be a real arbitrary Banach space and $\mathrm{K}$ be a nonempty closed bounded and convex subset of $\mathrm{X} . T_{i}$ local strongly pseudo contractive self mapping of $\mathrm{k}$ and uniformly continuous such that $F(T) \neq \varphi$ and the sequence $\left\langle x_{n}>\right.$ iteratively for $x_{1} \in k$ defined by

$$
x_{n+1}=a_{n} x_{n}+b_{n} T y_{n}+c_{n} u_{n}
$$

$y_{n}=\hat{a}_{n} x_{n}+\dot{b}_{n} T z_{n}+\dot{c}_{n} v_{n}$

$z_{n}=\dot{a}_{n} x_{n}+\dot{b}_{n} T_{3} x_{n}+\dot{c}_{n} w_{n}$

Where $\left\langle u_{n} \geq\left\langle v_{n}\right\rangle\right.$ and $\left\langle w_{n}\right\rangle$ are bounded sequence in $\mathrm{K}$ and $\left.\left.<a_{n}\right\rangle,\left\langle\dot{a}_{n}\right\rangle,\left\langle\dot{a}_{n}\right\rangle,\left\langle b_{n}\right\rangle,\left\langle\hat{b}_{n}\right\rangle,\left\langle\hat{b}_{n}\right\rangle<c_{n}\right\rangle$,

$\left\langle\dot{c}_{n}\right\rangle$ and $\left\langle\dot{c}_{n}\right\rangle$ are real sequences in $[0,1]$ such that

$a_{n}+b_{n}+c_{n}=\dot{a}_{n}+\dot{b}_{n}+\dot{c}_{n}=\dot{a}_{n}+\dot{b}_{n}+\dot{c}_{n}=1$ and satisfying the following conditions:

1- $\sum b_{n}=\infty$

2- $\lim _{n \rightarrow \infty} b_{n}=\lim _{n \rightarrow \infty} \dot{b}_{n}=0=\lim _{n \rightarrow \infty} \ddot{b}_{n}=0$

3- $\propto_{n}=b_{n}+c_{n}, \beta_{n}=\dot{b}_{n}+\dot{c}_{n}, \gamma_{n}=\dot{b}_{n}+\dot{c}_{n}$ and $\lim _{\mathrm{n} \rightarrow \infty} \quad \frac{1}{1+k_{x} \propto_{n}}=0$

for all $k_{x} \in(0,1)$. Then the sequence $<x_{n}>$ converges strongly to the unique fixed point of $T_{i}$

Proof:-

If $\mathrm{T}_{1}=\mathrm{T}_{2}=\mathrm{T}_{3}=\mathrm{T}$ in theorem(2.3), then corollary(2.4) follows immediately.

\section{Remark (2.6):-}

Theorems (2.1) and (2.2) are generalization of Yuguang and Fang [12] and Rafiq [13] via replace Mann iteration process with errors for strongly pseudo contractive (strongly accretive) by more general modified two-step iterative with errors for local strongly pseudo contractive (local strongly accretive) operators respectively.

\section{Remark (2.7):-}

Theorems (2.3) and (2.5) are generalization of

1- Results of Noor [4] and Imoru [10], [11] via replace three-step iteration of strongly pseudo contractive (strongly accretive) by a more general modified three-step iterative of local strongly pseudo contractive (local strongly accretive) respectively.

2- Results of Xue and Fan[8] and Rafiq [5] via replace modified three-step iterative scheme by a more general modified three-step iterative scheme with errors of local strongly pseudo contractive (strongly accretive) operators.

3- Results of mohbademu and Olalean[9]. via replace strongly pseudo contractive (local strongly accretive) by local strongly pseudo contractive (local strongly accretive) operators respectively. 


\section{Theorem (2.5):-}

Let $\mathrm{X}$ be a real arbitrary Banach space and $\mathrm{K}$ be a nonempty closed bounded and convex subset of $\mathrm{Z}$

$T_{i}$ is local strongly accretive self mapping of $\mathrm{K}(\mathrm{i}=1,2,3)$, uniformly continuous and $\bigcap_{i=1}^{3} F\left(T_{i}\right) \neq \emptyset$. define a mapping $R_{i}: k \rightarrow k$ by $R_{i} x=x-T_{i} x+f$, for some $f \in X$, consider the sequence defined by:

For arbitrary $x_{1} \in \mathrm{K}$

$$
\begin{aligned}
& x_{n+1}=a_{n} x_{n}+b_{n} R_{1} y_{n}+c_{n} u_{n} \\
& y_{n}=a_{n} x_{n}+\dot{b}_{n} R_{2} z_{n}+\dot{c}_{n} v_{n} \\
& z_{n}=\dot{a}_{n} x_{n}+\dot{b}_{n} R_{3} x_{n}+\dot{c}_{n} w_{n}
\end{aligned}
$$

Where $\left\langle u_{n} \geq\left\langle v_{n}\right\rangle\right.$ and $\left\langle w_{n}\right\rangle$ are bounded sequence in $\mathrm{K}$ and $\left\langle a_{n}\right\rangle,\left\langle\dot{a}_{n}\right\rangle,\left\langle\hat{a}_{n}\right\rangle,\left\langle b_{n}\right\rangle,\left\langle\hat{b}_{n}\right\rangle,\left\langle\hat{b}_{n}\right\rangle\left\langle c_{n}\right\rangle$,

$<\dot{c}_{n}>$ and $\left\langle\dot{c}_{n}>\right.$ are real sequences in $[0,1]$ such that

$a_{n}+b_{n}+c_{n}=\dot{a}_{n}+\dot{b}_{n}+\dot{c}_{n}=\dot{a}_{n}+\hat{b}_{n}+\dot{c}_{n}=1$ and satisfying the following conditions:

1- $\sum b_{n}=\infty$

2- $\lim _{n \rightarrow \infty} b_{n}=\lim _{n \rightarrow \infty} \hat{b}_{n}=\lim _{n \rightarrow \infty} \stackrel{\prime}{b}_{n}=0$

3- $\propto_{n}=b_{n}+c_{n}, \beta_{n}=\dot{b}_{n}+\dot{c}_{n}, \gamma_{n}=\dot{b}_{n}+\dot{c}_{n}$ and $\lim _{n \rightarrow \infty} \frac{1}{1+k_{x} \propto_{n}}=0$

For all $k_{x} \in(0,1)$. Then the sequence $<x_{n}>$ converges strongly to the unique solution of the equation $T_{i} x=f$.

\section{Proof:-}

It follows from definition of local strongly accretive mappings, that for given, there exists $k_{x} \in(0,1)$. such that

$$
<T_{i} x-T_{i} y, j(x-y)>\geq k_{x}\|x-y\|^{2} \text { for all } y \in X
$$

We observe that $R_{i}, T_{i}$ are uniformly continuous and for any given $f \in k$.

$$
\left(I-R_{i}\right) x=x-f+T_{i} x-x=T_{i} x-f
$$

Which implies that

$$
<\left(I-R_{i}\right) x-\left(I-R_{i}\right) y, j(x-y)>\geq k_{x}\|x-y\|^{2}
$$

That is $\left(I-R_{i}\right)$ is local strongly accretive. Thus $R_{i}$ is local strongly pseudo contractive. thus theorem(2.5) follows from theorem(2.3)

\section{References}

[1] Liu Z. , Kang S.M. and Shim S.H. " Almost stability of the Mann iteration method with errors for strictly Hemi-contractive operators in smooth Banach spaces" Korean Math S.C. 40(2003) pp.(22-40)

[2] Gurudwan. N. and Sharma. B.K. "convergence theorems for the zeros of a finite family of Generalized $\varphi$-accretive operators" J.Non linear sci Appl. 2(2009) pp.(260-269).

[3] Mogbademu A.A. and Olalean J.O. "modified Noor iterative methods for a family of strongly pseudo contractive maps" Bull of Math. Appl. 2(2011). Pp0(132-139).

[4] Noor M.A. , Rassia T.M. and Huang .Z. "three step iteration for Non linear accretive operators equations" J. Math .Aual. Appl. 274(2002) pp.(59-68). 
[5] Owojori O. O. and Imoru c.o "A general Ishikawa iteration sequence for non linear uniformly continuous pseudo- contractive operators in Arbitrary Banach spaces" proc. Jung. Math. Soc, 4(2002) pp.(91-100).

[6] Owojori O. O. and Imoru c.o "New iteration methods for pseudo- contractive and accretive operators in Arbitrary Banach spaces" krag.J. Math, 25(2003) pp.(97-110).

[7] Rafiq .A. "Modified Noor iterations for non linear equations in Banach spaces", Appl. Math. Comput. 182(2006) pp.(589-595).

[8] Rafiq .A. "Mann. Accretive scheme for non linear equations", Math. Comun. 12(2007) pp.(25-31).

[9] Xue Z. and Fan. R. "some comments on Noor's iterations is Banach spaces", Appl. Math. Comput. 206(2008) pp.(12-15).

[10] Yany L. "the equivalence of Ishikawa Mann and Multi-self iterations is Banach spaces", A Joural of Chinese univ., 16(2007) pp.(83-91).

[11] Yugang. Xu, Fang XIE. "Stability of Mann iterations process with Random errors for the fixed point of strongly pseudo contractive mapping in Arbitrary Banach spaces", math. Kolloq 58(2004) pp.(93-100).

[12] Zeqing, L., Yuguang, $X$ and shim. K.M."almost stable iterations schemes for local strongly pseudo contractive and local strongly accretive operators in real uniformly smooth Banach spaces" Aeta. Math , 2(2008). Pp.(285-298). 